\title{
Influencia de variables individuales y de contexto en el aprendizaje al finalizar la primaria
}

Influence of individual and contextual, variables on learning at the end of the primary

\section{Volumen 19, Número 3 \\ Setiembre-Diciembre \\ pp. 1-19}

\author{
Aldo Bazán-Ramírez \\ Eduardo Hernández-Padilla \\ Doris Castellanos-Símons
}

Citar este documento según modelo APA

Bazán-Ramírez, Aldo., Hernández-Padilla., Eduardo y Castellanos-Símons, Doris. (2019). Influencia de variables individuales y de contexto en el aprendizaje al finalizar la primaria. Revista Actualidades Investigativas en Educación, 19(3), 1-19. Doi. 10.15517/aie.v19i3.38644 


\title{
Influencia de variables individuales y de contexto en el aprendizaje al finalizar la primaria \\ Influence of individual and contextual, variables on learning at the end of the primary
}

\author{
Aldo Bazán-Ramírez \\ Eduardo Hernández-Padilla² \\ Doris Castellanos-Símons ${ }^{3}$
}

\begin{abstract}
Resumen: Diversos estudios nacionales e internacionales han mostrado que las variaciones en el aprendizaje del estudiantado mexicano se encuentran asociadas a variables de tipo socioeconómico, personal y contextual entre otras. En el presente artículo, mediante el modelamiento jerárquico multinivel, se analizó el efecto de variables personales y de contexto en el aprendizaje de las competencias Matemáticas y Comprensión lectora en el alumnado de sexto grado de primaria, proveniente de dos distintos contextos educativos: escuela privada y pública-rural indígena. Asimismo, se incluyeron variables de la persona educanda, tales como edad, sexo, grado escolar, entre otras, en los análisis. Los resultados evidencian que, cuando se controlaron los efectos del contexto de procedencia, el desempeño educativo en comprensión lectora y en matemáticas, fueron explicados, en mayor medida, por las diferencias individuales $y$, cuando se controlaron las diferencias individuales, la variabilidad en el aprendizaje se asoció al contexto de procedencia. Los resultados también corroboraron los hallazgos de otros trabajos donde se evidenció que el tipo de sostenimiento de la escuela es una variable que influyó en el logro: la población de las escuelas privadas procedió en su mayor parte, de hogares con elevado nivel socioeconómico cultural, respecto del alumnado que recibe el servicio público-rural-indígena.
\end{abstract}

Palabras clave: aprendizaje; competencias matemáticas; comprensión lectora; contexto de aprendizaje, educación primaria.

\begin{abstract}
Several national and international studies have shown that the variations in the learning of the Mexican student body are associated to socioeconomic, personal and contextual variables among others. In the present work, through the multilevel hierarchical modeling, the effect of the personal and context variables in the learning of the mathematical competences and reading comprehension in the sixth-grade students from two different educational contexts (private school and indigenous public-rural). Likewise, personal variables of the student, such as age, sex, grade level, etc., were included in the analysis. The results obtained show that, controlling the effect of the context of origin, the educational performance in Reading Comprehension and in Mathematics, are explained to a greater extent by the individual differences; and, when individual differences were controlled, the variability in learning is sensitive to the context of origin. The results obtained confirm the findings of other studies which show that the type of school support is a variable that influences achievement: the population of private schools comes, for the most part, from homes with a high socio-economic level, attend come from a socioeconomic level above its counterpart that receives the public-rural-indigenous service.
\end{abstract}

Keywords: learning; mathematics competences; reading comprehension; school context of provenance, primary education.

\footnotetext{
1 Profesor Investigador de la Universidad Autónoma del Estado de Morelos, México. Dirección electrónica: abazanramirez@gmail.com

2 Profesor Investigador de la Universidad Autónoma del Estado de Morelos, México. Dirección electrónica: eduhpad@gmail.com

3 Profesora Investigadora de la Universidad Autónoma del Estado de Morelos, México. Dirección electrónica: dcastellanos@uaem.mx
}

Artículo recibido: 5 de abril, 2019

Enviado a corrección: 11 de junio, 2019

Aprobado: 15 de julio, 2019 


\section{Introducción}

Pese a las continuas reformas educativas y a las diversas modificaciones de los planes y programas de estudio en la educación básica en México desde los años 90, los resultados educativos a mediados de las primeras décadas del siglo XXI mostraban no solo bajos indicadores de aprovechamiento, sino también indicios de desigualdad de acuerdo con el contexto socioeconómico de procedencia de la comunidad estudiantil. Dichos resultados mostraron diferencias sustanciales entre los desempeños que obtiene el estudiantado de alto nivel económico y los obtenidos por sus contrapartes que proceden de sectores desfavorecidos; tales resultados evidenciaron que, en la educación básica mexicana, el contexto educativo es un predictor importante del desempeño escolar (Blanco, 2017; Carvallo, 2006; Carvallo, Caso y Contreras, 2007; Vázquez-Chagoyán, 2005).

Los resultados educativos en diferentes evaluaciones aún reflejan que el alumnado de contextos rurales y de los sistemas de educación indígena obtiene los peores indicadores de logro educativo, en contraste con el alumnado de escuelas privadas, que obtienen mejores indicadores de logro académico. Estos resultados educativos se deben, más bien, a las diferencias en las oportunidades educativas, a las diferencias en las condiciones instruccionales de aprendizaje y a las brechas socioeconómicas y de capital cultural del origen del estudiantado.

Las evaluaciones nacionales sobre desempeño académico en educación básica en México, con la prueba de Evaluación Nacional del Logro Académico en Centros Escolares (ENLACE), mostraron que las escuelas Privadas obtuvieron resultados significativamente superiores a las escuelas Indígenas, Generales y CONAFE, en el tercer, cuarto, quinto y sexto de primaria (Secretaría de Educación Pública [SEP], 2013), y reflejaban un panorama más general y a la vez diferenciado de los resultados de aprendizaje por contexto, en indicadores de logro por asignaturas, y los niveles de logro alcanzados en cada asignatura. Asimismo, la aplicación del Plan Nacional para la Evaluación de los Aprendizajes (PLANEA) entre 2015 y 2018, también evidenció estos resultados desiguales tomando en cuenta el contexto de procedencia (Instituto Nacional para la Evaluación de la Educación [INEE], 2016).

En ambos casos, los indicadores más bajos corresponden al alumnado que procede de contextos sociodemográficos más desfavorecidos, principalmente de escuelas indígenas y comunitarias de las zonas rurales, mientras que el de escuelas privadas, es el que obtiene mejores resultados en dichas evaluaciones. 
El objetivo de este estudio fue someter a prueba dos modelos jerárquicos multinivel para explicar el desempeño académico en Comprensión Lectora y en Matemáticas del estudiantado que había concluido el 6to grado de primaria y que proviene de dos contextos socioeconómicos: instituciones privadas de estatus económico alto e instituciones públicas de zonas rurales de población indígena, en Morelos y Sonora, México. Morelos es un Estado de la región centro sur de la República Mexicana y Sonora es un Estado de la región noroeste.

Los modelos jerárquicos incluyen un modelo nulo (intercepto), un modelo predictivo de variables de estudiantes (edad, sexo, grado escolar, calificación del maestro), escuela (grupo y escuela de procedencia), familia (grado escolar del padre o de la madre; vive con ambos padres, otro adulto con quien vive; número de horas en que la madre trabaja fuera del hogar) y un modelo de contextos (contexto de procedencia).

En Morelos, la muestra de estudiantes del contexto rural indígena proviene de la localidad de Xoxocotla, Municipio de Puente de Ixtla, de la localidad de Cuentepec del Municipio de Temixco, al sur del Estado. En Sonora, la muestra de estudiantes del contexto rural indígena proviene de las localidades de Loma de Bacum y Loma de Guamuchil en la Región Yaqui, en el sur del Estado de Sonora. La escuela privada en Morelos está ubicada en la zona norte del Municipio de Cuernavaca, y la escuela privada en Sonora está ubicada en la zona norponiente del Municipio de Cajeme, Ciudad Obregón.

Este trabajo fue realizado en el marco de un proyecto de investigación financiado por el Consejo Nacional de Ciencia y Tecnología de México (CONACYT), cuya vigencia fue del 15 de marzo de 2012 al 14 de marzo de 2016. La investigación fue realizada en el periodo comprendido entre junio de 2014 y diciembre de 2015.

\section{Referente teórico}

El contexto de procedencia del alumnado (condiciones económicas, culturales y acceso a la salud, la educación y los recursos tecnológicos de las familias de origen), así como el contexto de su comunidad influyen en el aprendizaje y en el logro académico. Desde el famoso informe Coleman (Coleman, et. al, 1966) se han realizado diversos estudios e informes en los cuales, el contexto de origen del estudiantado explica de forma significativa el logro educativo.

Estudios con evaluaciones a gran escala en México y en otros países latinoamericanos respecto a resultados de aprendizaje, medidos como logro educativo, por 
ejemplo, las pruebas Programme for International Student Assessment (PISA); Exámenes para la Calidad y el Logro Educativo (EXCALE), y las evaluaciones Segundo Estudio Regional Comparativo y Explicativo (SERCE) y Tercer Estudio Regional Comparativo y Explicativo (TERCE) han evidenciado que el contexto de procedencia del alumnado es un predictor importante del desempeño escolar, y que a mayor nivel socioeconómico se tiene un mejor indicador de logro educativo, debido a que el estudiantado de los contextos más favorecidos disponen de mayores oportunidades de aprendizaje y de capital cultural (Alanís, Ávila y Lerma, 2009; Blanco, 2017; Backhoff, 2011; Backhoff, Andrade, Peón, Sánchez y Bouzas, 2006; Backhoff, Sánchez, Peón y Andrade, 2010; Backhoff, Andrade, Sánchez y Peón, 2008; Cervini, 2002).

Sin embargo, se debe tomar en cuenta que el contexto de procedencia por sí mismo no explica de forma directa el logro educativo individual, puesto que diversas escuelas o algunos o algunas estudiantes de contextos sociodemográficos pobres o desfavorecidos, pueden presentar altos indicadores de logro educativo, y escuelas o el alumnado de contextos socioeconómicos altos podrían presentar bajos indicadores de logro educativo, siendo factores relevantes para el desempeño educativo; el apoyo, las expectativas educativas y el interés de la familia (Bazán, Urbina, Domínguez, Mansillas y Gómez, 2011; Castro, Lubker, Bryant y Skinner, 2002; Mella y Ortíz, 1999; Reese, Mejía y Ray, 2012).

En este estudio se utiliza el análisis jerárquico multinivel. Esta metodología de análisis de regresión está basada en la concepción de que las personas que conforman una muestra están agrupadas y anidadas respecto a un grupo en particular, el cual está conformado por personas que comparten algún rasgo distintivo o una zona geográfica común. La agrupación en una zona geográfica específica sugiere efectos de variables a nivel individual y grupal sobre una variable dependiente, efectos que, en un análisis de regresión lineal simple, no se verían explícitamente reflejadas; cuya significatividad formal debe ser igual o menor a 0,05.

Los modelos de regresión multinivel asumen que los datos están organizados por estratos con solo una variable dependiente en el nivel de alumnos y distintas variables explicativas en diversos niveles, y que pueden variar aleatoriamente en el segundo nivel. Un ejemplo es la siguiente fórmula $y_{i j}=\beta_{0}+\beta_{1} x_{i j}+u_{j}+e_{i j}$ donde se muestra una variable dependiente $y_{i j}$, el valor del intercepto en el segundo nivel y su componente de error, $\beta_{0}$ y $e_{i j}$, respectivamente; la pendiente de una variable independiente $\beta_{1} x_{i j}$ y su componente de error $u_{j}$. 
En el contexto mexicano se han reportado diversos estudios utilizando modelos jerárquico multinivel para estimar el efecto de variables a nivel individual, a nivel escuela, contexto sociocultural, etc., sobre indicadores de logro académico en diversos niveles y contextos educativos (Blanco, 2017; Carvallo, et al., 2007; Hernández y Bazán, 2016; Hernández, 2018). Asimismo, Cueto, León, Muñoz y Rosales (2016) ha reportado el uso de análisis jerárquico multinivel para explicar el efecto de diversas variables predictoras sobre el logro en lectura de estudiantes de Perú en la prueba PISA 2009.

\section{Método}

\subsection{Enfoque}

El enfoque de esta investigación fue cuantitativo, con un diseño transeccional predictivo estratificado en dos niveles, y alcance explicativo. Esto último debido, por una parte, a la naturaleza de las variables que se incluyen en los análisis, y por la otra, al tipo de análisis basado en la regresión lineal.

\subsection{Población de estudio}

Participaron 300 estudiantes, seleccionados intencionalmente, que habían concluido el sexto grado de primaria, 98 procedentes del contexto de escuelas privadas y 202 de escuelas públicas del contexto rural-indígena. El criterio de inclusión para el alumnado fue, estar en el último bimestre del sexto de primaria o en el primer bimestre de primero de secundaria.

Para el contexto rural indígena, en el Estado de Morelos, se incluyeron dos secciones de 6to grado de una escuela primaria pública y dos secciones de primer año de una Telesecundaria. En el Estado de Sonora se incluyeron dos secciones de primer año de secundaria por cada una de dos Telesecundarias. Para el contexto privado, fueron incluidas dos secciones de primer año de secundaria de un colegio particular por cada Estado.

Las escuelas fueron seleccionadas por conveniencia, con la finalidad de que sea representativa, dos instituciones escolares por cada contexto en cada Estado; sin embargo, solo en el contexto rural indígena se pudo cumplir con este criterio, porque en el contexto privado, dada las condiciones de inseguridad pública, fue difícil obtener el permiso de las escuelas privadas, y solo se contó con una escuela privada por Estado.

El consentimiento informado se obtuvo de la persona adulta responsable de cada estudiante en reuniones realizadas con padres de familia en los ambientes de la propia 
escuela. Asimismo, las personas que participaron en el estudio proporcionaron su asentimiento mediante un formato breve impreso.

\subsection{Técnicas de recolección}

La información fue recopilada mediante la aplicación de dos instrumentos de evaluación de aprendizajes y un cuestionario sociodemográfico. Las primeras aplicaciones se llevaron a cabo en el mes de junio de 2015, en dos primarias en el Estado de Morelos. A finales de agosto e inicios de septiembre de 2015, se llevó a cabo la segunda aplicación en Morelos y la aplicación en Sonora, de los instrumentos de evaluación de aprendizajes en español y en matemáticas de sexto grado de primaria.

La razón por la cual hubo dos periodos de toma de datos se debió principalmente al pedido de algunas escuelas debido a la cercanía del periodo vacacional, o debido a que algunas secundarias solicitaron una valoración de sus estudiantes que iniciaban la secundaria, respecto a las competencias en español y matemáticas que habían desarrollado en el sexto grado de la primaria. En Sonora, un motivo adicional y fundamental es que la finalización del ciclo escolar se adelanta un mes debido a las condiciones climatológicas.

En una primera sesión se evaluó la comprensión lectora, entre 40 y 60 minutos. Al siguiente día se aplicó la evaluación de matemáticas, en una sesión entre 60 y 80 minutos. Al término de la aplicación, los evaluadores recogían los instrumentos y se cercioraban de que todos estuvieran contestados. A partir de fines de agosto de 2015, se volvió a contactar a las diferentes escuelas para solicitarles las calificaciones finales en Español, en Matemáticas y los promedios generales obtenidos por los alumnos en el sexto grado de primaria a partir de sus boletas y/o registros de los participantes.

Fueron utilizados los siguientes instrumentos de medición:

Evaluación del logro en Comprensión lectora. Conformado por 10 tareas que evalúan la competencia desarrollada en la asignatura de Español de sexto grado de primaria, con cinco temas de reflexión en común (ver Tabla 1). La respuesta de cada estudiante es calificada en una escala del 0 al 4, según el cumplimiento del criterio de logro establecido por la tarea, no existen respuestas incorrectas, solo valores de logro en rangos cualitativamente jerarquizados. 
Tabla 1. Aprendizajes esperados de 6to de primaria en la competencia Comprensión Lectora, al egreso de la primaria en el ciclo escolar 2014-2015

\begin{tabular}{ll}
\hline Aprendizajes esperados & Ítems \\
\hline Lectura de biografías & $\begin{array}{c}\text { BiogLec1 } \\
\text { BiogLect2 }\end{array}$ \\
\hline Lectura de cuentos de misterio y terror & $\begin{array}{c}\text { TerrLec1 } \\
\text { TerrLec2 }\end{array}$ \\
\hline Lectura de sucesos históricos & ZapLec1 \\
& ZapLec2 \\
\hline Lectura de cartas & CartLec1 \\
& CartLec2 \\
\hline Lectura de poemas & PoemLec1 \\
& PoemLec2
\end{tabular}

Fuente. Elaboración propia.

Nota. Se presentan los códigos de identificación de los diferentes reactivos.

La validez de constructo de este instrumento, fue obtenida mediante un Análisis Factorial Confirmatorio con modelamiento de ecuaciones estructurales, utilizando el programa EQS 6,4. La competencia Comprensión Lectora fue conformada por cinco constructos (los cinco aprendizajes esperados mostrados en la Tabla 1), y cada constructo o variable manifiesta incluyó dos indicadores o también llamadas variables manifiestas (las tareas o ítems).

Se obtuvo un modelo con buena bondad de ajuste (Chi cuadrada $=36,25$; $P=0,01$; $\mathrm{CFI}=0,99$; RMSEA $=0,04)$ y un coeficiente alfa de Cronbach $=0,80$ con datos ordinales. Los pesos factoriales entre constructos e ítems variaron entre 0,44 y 0,85, y las covarianzas entre los cinco constructos de aprendizajes esperados de comprensión lectora variaron entre 0,47 (covarianza moderada) y 0,79 (covarianza alta).

De acuerdo con estos datos, se tiene un instrumento con una aceptable validez convergente y divergente de constructo, y en consecuencia, puede obtenerse un indicador general de la competencia Comprensión lectora.

Evaluación de Competencias matemáticas. Es un instrumento de 16 tareas, que evalúa las cuatro competencias establecidas a desarrollar en el sexto grado de primaria; tiene ocho temas de reflexión en común los cuales también son denominados Aprendizaje Esperado (ver Tabla 2). Cada respuesta del estudiante fue calificada en una escala del 0 al 4, según el cumplimiento del criterio de logro establecido por la tarea, no existen respuestas incorrectas, solo valores de logro en rangos cualitativamente jerarquizados. 
Tabla 2. Aprendizajes esperados en Matemáticas de 6to de primaria, por competencias, al egreso de la primaria en el ciclo escolar 2014-2015

\begin{tabular}{|c|c|c|}
\hline Competencia & Dimensión (Aprendizaje esperado) & Ítems \\
\hline \multirow{2}{*}{$\begin{array}{l}\text { Comunicar } \\
\text { información } \\
\text { matemática }\end{array}$} & $\begin{array}{l}\text { Interpretar la información matemática } \\
\text { contenida en las gráficas }\end{array}$ & $\begin{array}{l}\text { CIM1Int } \\
\text { CIM2Int }\end{array}$ \\
\hline & $\begin{array}{l}\text { Deducir la información matemática contenida } \\
\text { en las gráficas }\end{array}$ & $\begin{array}{l}\text { CIM2Ded } \\
\text { CIM1Ded }\end{array}$ \\
\hline \multirow{2}{*}{$\begin{array}{l}\text { Validar } \\
\text { procedimientos y } \\
\text { resultados }\end{array}$} & $\begin{array}{l}\text { Expresar el procedimiento utilizado para } \\
\text { resolver un problema matemático }\end{array}$ & $\begin{array}{l}\text { VPR1Exp } \\
\text { VPR2Exp }\end{array}$ \\
\hline & $\begin{array}{l}\text { Argumentar el procedimiento utilizado para } \\
\text { resolver un problema matemático }\end{array}$ & $\begin{array}{l}\text { VPR2Arg } \\
\text { VPR1Arg }\end{array}$ \\
\hline \multirow{2}{*}{$\begin{array}{l}\text { Manejar técnicas } \\
\text { eficientemente }\end{array}$} & $\begin{array}{l}\text { Identificar el procedimiento adecuado para } \\
\text { resolver un problema matemático }\end{array}$ & $\begin{array}{l}\text { MTE1Ele } \\
\text { MTE2Ele }\end{array}$ \\
\hline & $\begin{array}{l}\text { Plantear un procedimiento alterno para } \\
\text { resolver un problema matemático }\end{array}$ & $\begin{array}{l}\text { MTE2Usa } \\
\text { MTE1Usa }\end{array}$ \\
\hline \multirow{2}{*}{$\begin{array}{l}\text { Resolver } \\
\text { problemas de } \\
\text { manera autónoma }\end{array}$} & $\begin{array}{l}\text { Elegir los datos necesarios para resolver un } \\
\text { problema matemático }\end{array}$ & $\begin{array}{l}\text { RPMA1lde } \\
\text { RPM2Alde }\end{array}$ \\
\hline & $\begin{array}{l}\text { Emplear un procedimiento alterno para } \\
\text { resolver un problema matemático }\end{array}$ & $\begin{array}{l}\text { RPMA2Pla } \\
\text { RPMA1Pla }\end{array}$ \\
\hline
\end{tabular}

Fuente: Elaboración propia

En la obtención de la validez convergente y divergente de la escala para evaluar este constructo fue sometida a prueba un modelo de análisis factorial confirmatorio (AFC) de segundo orden (segundo nivel) y se obtuvo una moderada validez convergente y divergente de constructo (Chi cuadrada $=447,92 ; \mathrm{P}=0,00 ; \mathrm{CFI}=0,89$; $\mathrm{RMSEA}=0,08$ ).

Este modelo está representado desde dos niveles. El segundo nivel es la competencia, conformada por cuatro constructos o variables latentes: Comunicar información matemática, Validar procedimientos y resultados, Manejar técnicas eficientemente y Resolver problemas de manera autónoma. El primer nivel está conformado por los constructos de aprendizaje esperado, por ejemplo, Interpretar la información y, Deducir información, para el caso de la primera competencia. En este primer nivel se incluyó dos aprendizajes esperados (constructos) por cada competencia, en total, ocho constructos de primer nivel, y cada constructo de aprendizaje esperado incluyó dos indicadores o ítems.

En el primer nivel, los pesos factoriales entre constructos aprendizajes esperados e ítems, variaron entre 0,48 y 0,83; en tanto que en el segundo nivel, las cargas factoriales entre constructos de competencia y constructos de aprendizajes esperado, variaron entre 
0,78 y 0,99 . Por su parte, las covarianzas de los constructos de segundo orden (competencias) oscilaron entre 0,58 y 0,97. Asimismo, el modelo mostró un coeficiente Alfa de Cronbach $=0,86$. Con base en los resultados, se puede tomar un solo atributo de logro en matemáticas con cuatro índices directos, uno por cada competencia evaluada, para estimar el desempeño del alumnado.

Ficha sociodemográfica. Mediante autoreportes de cada estudiante se obtuvieron información acerca de variables personales y de contexto familiar, como: Vive con la mamá y/o el papá, El adulto o adulta con quien vive, Número de horas que la madre o tutora trabaja fuera de la casa, etc. De igual forma, en los autoreportes se incluyó información del alumnado a partir de datos administrativos de las escuelas: Edad, Sexo, Grado y grupo o sección, Calificaciones en Español, Calificaciones en Matemáticas, Contexto sociodemográfico de procedencia (Privado o Público, Status socio económico).

\subsection{Procesamiento de análisis}

Mediante el Software estadístico Winstep HLMC, fueron realizados dos análisis jerárquicos multinivel, uno para comprensión lectora, y otro para matemáticas. En la Tabla 3 se presentan los códigos usados en los análisis multinivel y mostrados en las tablas de resultados, así como la mención de lo que significaron o representaron cada variable. En la misma, puede apreciarse que la mayoría de las variables fueron categóricas de nivel ordinal, y solo existen dos variables de nivel intervalar, como son la calificación en Comprensión lectora y Matemáticas (cada una compuesta por el promedio de las diferentes sub-escalas que la conforman: cinco para Comprensión lectora y cuatro para la competencia de Matemáticas).

La colinealidad, o la combinación lineal entre dos predictores de un modelo de regresión de tal forma que no logren distinguirse los efectos individuales de cada variable la variable entidad (Sonora y Morelos) no se introdujo; en este caso debido a los efectos de colinearidad (es decir,), de que un tipo de contexto sea exclusivo de una de las entidades federativas. 
Tabla 3. Códigos y significados de las variables incluidas en el análisis jerárquico multinivel para las variables dependientes Comprensión lectora y competencias en Matemáticas, en el ciclo escolar 2014-2015

\begin{tabular}{ll}
\hline \multicolumn{1}{c}{ Código } & \multicolumn{1}{c}{ Significado } \\
\hline EDAD & Edad \\
SEXO & Sexo \\
GRADGRUP & Grado y grupo del estudiante \\
CALIFE & Calificaciones en Español \\
CALIFM & Calificaciones en Matemáticas \\
GRADOESC & Grado escolar del padre o madre (se tomó al de mayor grado de \\
VIVAMPA & escolaridad) \\
QUIVIVE & Vive con la mamá o el papá \\
HOTRAFUC & Con quien vive \\
PRIVADO & Número de horas que la madre o tutora trabaja fuera de la casa \\
RURAL & Contexto sociodemográfico de procedencia - Privado \\
& Contexto sociodemográfico de procedencia - Rural-Indígena \\
COMPRENSION LECTORA & $\begin{array}{l}\text { Puntaje total en la suma de las dos subescalas Comprensión lectora } \\
\text { y Producción de textos }\end{array}$ \\
MATEMATICAS & $\begin{array}{l}\text { Puntaje total en la suma de las dos subescalas: Comunicar } \\
\text { información matemática, Validar procedimientos y resultados, } \\
\text { Manejar técnicas eficientemente y Resolver problemas de manera } \\
\text { autónoma }\end{array}$ \\
\hline
\end{tabular}

Fuente: elaboración propia.

\section{Resultados}

En la Tabla 4 se muestran los resultados el análisis jerárquico multinivel para los resultados de Competencia lectora, donde se incluyen algunas variables de nivel individual y otras de nivel grupal.

Para el análisis fueron sugeridos dos modelos, además del modelo del intercepto. El primero de ellos se denomina modelo predictivo, el cual incluye las variables personales y escolares, así como y las calificaciones en Español y Matemáticas. Este modelo intenta predecir los resultados en Competencia lectora basándose en variables individuales y en las calificaciones escolares de Español y Matemáticas. El segundo, y último modelo, es denominado modelo contextos, ya que considera variables de contexto de procedencia del estudiante. La variable de referencia es Urbano-pública por cuanto el contexto de escuelas privadas y rurales indígenas es codificado para ser comprendido. Otra variable dicotómica fue sexo, donde el valor de referencia es masculino. 
Tabla 4. Resultado de la relación entre variables predictoras y logro en Comprensión Lectora en 6to de primaria, según tres modelos de Análisis Jerárquico Multinivel

\begin{tabular}{|c|c|c|c|c|c|c|}
\hline & \multicolumn{2}{|c|}{ Modelo Intercepto } & \multicolumn{2}{|c|}{ Modelo Predictivo } & \multicolumn{2}{|c|}{ Modelo Contextos } \\
\hline & $\begin{array}{l}\text { Coeficient } \\
\mathrm{e}\end{array}$ & (E.E.) & Coeficiente & (E.E.) & Coeficiente & (E.E.) \\
\hline Intercepto & 69.15 & $(11.23)$ & 31.57 & $(66.02)$ & 49.25 & $(69.07)$ \\
\hline Edad & & & 6.18 & $(05.25)$ & 3.31 & $(05.17)$ \\
\hline Sexo & & & 11.48 & $(05.86)$ & 12.30 & $(05.73)$ \\
\hline GradGrup & & & 0.53 & $(01.50)$ & 0.98 & $(01.49)$ \\
\hline GradoEsc & & & 3.92 & $(00.01)$ & -9.82 & $(08.16)$ \\
\hline VivaMPa & & & -10.43 & $(08.24)$ & 1.65 & $(01.73)$ \\
\hline QuiVive & & & 2.13 & $(01.72)$ & 2.92 & $(01.57)$ \\
\hline HoTraFuC & & & 0.87 & $(02.56)$ & 0.58 & $(02.50)$ \\
\hline CALIFE & & & 7.42 & $(03.48)$ & 7.74 & $(03.57)$ \\
\hline CALIFM & & & 10.90 & $(03.57)$ & 9.98 & $(03.72)$ \\
\hline PRIVADO & & & & & 47.31 & $(11.02)$ \\
\hline RURAL & & & & & --49.24 & $(14.44)$ \\
\hline \multicolumn{2}{|c|}{ Varianza Total } & $\begin{array}{l}1644.19 \\
2\end{array}$ & & 659.803 & & 532.83 \\
\hline \multicolumn{2}{|c|}{ Varianza escuela } & $\begin{array}{l}687.080 \\
6\end{array}$ & & 118.846 & & 0.7521 \\
\hline \multicolumn{2}{|c|}{ Varianza Individual } & $\begin{array}{l}957.111 \\
6\end{array}$ & & 540.957 & & 532.08 \\
\hline
\end{tabular}

Fuente: elaboración propia

Nota. Los resultados significativos de los coeficientes $(p<0.05)$, son mostrados en cursivas y en negritas.

Los resultados obtenidos muestran que la media de los sujetos de toda la muestra en la Competencia lectora es 69.15 puntos. Este modelo permite calcular la variabilidad explicada por las escuelas y por los individuos; en ese sentido, la varianza al nivel del estudiantado es significativamente grande y es el principal componente de la varianza total, lo que se confirma por la variabilidad relativamente baja que explican las escuelas, todo ello en el modelo de desempeño en la Competencia lectora.

En el Modelo Predictivo, las únicas variables independientes significativas son el Grado Escolar de los Padres o Madres y las Calificaciones que obtuvo el estudiante en las asignaturas de Español y de Matemáticas. La varianza total de dicho modelo es menor que el anterior, aunque la variabilidad al nivel de las escuelas mantiene un porcentaje reducido de la variabilidad para explicar los resultados. 
Finalmente, el Modelo Contextos tiene un intercepto positivo, pero que no es significativamente formal, esto demuestra que no existen diferencias relevantes para el estudiantado en torno a la media o intercepto de Competencia lectora. La inclusión de los Contextos Privado y Rural resulta en diferencias significativas asociadas con un valor negativo para el coeficiente del Contexto Rural, en tanto que del Privado asumió un valor positivo; este resultado coincide con varios hallazgos reportados en las evaluaciones a gran escala a nivel nacional e internacional. Por otro lado, tanto la influencia de las Calificaciones como la variable Sexo resultan estadísticamente significativas. El Sexo es particularmente relevante debido a que cuando en el Modelo se incluye el Contexto de Procedencia en los análisis predictivos, las Mujeres en promedio obtuvieron 10 puntos por encima de la calificación de los Hombres en Competencia lectora, siendo un resultado formalmente significativo.

El análisis multinivel de la Competencia Matemáticas se muestra en la Tabla 5; en dicha tabla se analizaron los resultados de Matemáticas en Sexto Grado, las variables que participaron en los modelos son las mismas que en el caso de la Competencia lectora. Nuevamente, los análisis realizados consistieron en tres modelos: el Modelo del Intercepto, que sirve de base para realizar comparaciones a nivel de la varianza del nivel del alumnado y el de las escuelas; el Modelo Predictivo, y el Modelo de Contextos, ambos explicados previamente.

Tabla 5. Resultado de la relación entre variables predictoras y logro en Matemáticas en 6to de primaria, según tres modelos de Análisis Jerárquico Multinivel

\begin{tabular}{|l|c|c|c|c|r|r|}
\hline & \multicolumn{2}{|c|}{ Modelo Intercepto } & \multicolumn{2}{c|}{ Modelo Predictivo } & \multicolumn{2}{c|}{ Modelo Contextos } \\
\hline & Coeficiente & $($ E.E.) & Coeficiente & $($ E.E.) & Coeficiente & $($ E.E.) \\
\hline Intercepto & $\mathbf{2 7 . 5 6}$ & $(04.71)$ & 77.90 & $(48.82)$ & $\mathbf{1 1 1 . 6 4}$ & $(51.14)$ \\
\hline Edad & & & -2.41 & $(03.77)$ & -3.13 & $(03.75)$ \\
\hline Sexo & & & 0.31 & $(03.76)$ & 0.80 & $(03.75)$ \\
\hline GradGrup & & & -1.97 & $(01.02)$ & $\mathbf{- 2 . 1 2}$ & $(01.03)$ \\
\hline GradoEsc & & & $-\mathbf{1 . 9 9}$ & $(00.95)$ & $\mathbf{- 2 . 6 8}$ & $(01.01)$ \\
\hline VivaMPa & & -6.44 & $(05.37)$ & -6.13 & $(05.35)$ \\
\hline QuiVive & & & 0.16 & $(01.11)$ & -0.11 & $(01.11)$ \\
\hline HoTraFuC & & 1.95 & $(01.61)$ & 1.83 & $(01.61)$ \\
\hline CALIFE & & -2.28 & $(02.32)$ & -2.15 & $(02.32)$ \\
\hline CALIFM & & $\mathbf{4 . 6 9}$ & $(02.36)$ & 4.20 & $(02.37)$ \\
\hline PRIVADO & & & & & 17.90 & $(12.73)$ \\
\hline RURAL & & & & 359.144 & & $(13.72)$ \\
\hline Varianza Total & & & 138.269 & & 283.83 \\
\hline Varianza escuela & 360.5958 & & 220.875 & & 21.05 \\
\hline Varianza Individual & 113.5015 & & & & & \\
\hline
\end{tabular}

Fuente: elaboración propia

Nota. Los resultados significativos de los coeficientes $(p<0.05)$ son mostrados en cursivas y negritas. 
El Modelo del Intercepto muestra que el valor de este último es relativamente bajo, aunque estadísticamente significativo. La varianza explicada al nivel de las escuelas en la Competencia matemáticas es, claramente, mucho menor que la exhibida en Competencia lectora. Sin embargo, el tamaño de la varianza individual, la del alumnado, es profusamente más grande: prácticamente el doble de variación de lo que puede explicar la escuela.

El Modelo Predictivo muestra que pese a tener un intercepto mayor que el modelo previo, este no es estadísticamente significativo puesto que de las variables consideradas para el esquema multinivel solamente dos de ellas resultaron significativas; tanto Grado Escolar del Padre o la Madre al igual que Calificación Promedio en Matemáticas mostraron una asociación positiva con Competencia matemáticas. Esto significatividad de variables no modifica la varianza total del Modelo del Intercepto, donde hay un incremento en la variabilidad entre escuelas y una disminución de la misma al nivel del alumnado, lo que sugiere que hay un mayor poder de explicación de la variabilidad gracias a algunas de las características analizadas de la escuela.

Finalmente, el Modelo Contextos, tiene un intercepto mayor y estadísticamente significativo; sin embargo, del grupo de variables individuales que son significativas en el aporte del modelo, en el Grado que cursa el estudiante y en la Escolaridad de los Padres predijeron significativamente, pero de manera negativa, el logro en matemáticas. La Calificación Escolar de matemáticas deja de ser significativa y el único contexto que resulta significativo es el Rural-Indígena, que tiene un valor negativo, lo cual indica que el estudiantado procedente de este contexto reporta en promedio valores por debajo de los obtenidos del alumnado de los contextos urbano y privado. La varianza total de este tercer Modelo (Contextos) muestra una disminución significativa que se presenta claramente en la variabilidad de las escuelas, debido a que la consideración del Contexto en el modelo es mayor que el de las escuelas. Finalmente, la varianza al nivel del estudiantado muestra cambios marginales.

En general, aunque los principales resultados obtenidos destacan lo señalado en otras investigaciones respecto a la potencial ventaja o superioridad de las escuelas privadas sobre las escuelas rurales e indígenas (Backhoff, et al., 2006; Backhoff, et al., 2008; Backhoff, et al., 2010; Carvallo, 2006; Carvallo, et al., 2007), cabe señalar que en el presente trabajo las calificaciones en las asignaturas de Español y Matemáticas juegan un papel muy importante en la predicción de los resultados obtenidos. Si bien es cierto que la asignatura de Español 
es sumamente permeable a los efectos socioeconómicos y culturales, el rendimiento en Matemáticas depende casi enteramente de lo que ocurre en el aula.

El impacto del llamado capital cultural y el contexto (y/o tipo de escuela) se traduce en mayores oportunidades de acceso a las influencias culturales, mejores oportunidades de aprendizaje y de un contexto facilitador (SEP, 2006), incluso para el propio desarrollo de las capacidades y aptitudes, lo que sugeriría, concretamente, la importancia de mayor estimulación de habilidades y competencias que están en su base y en su desarrollo. Para Gagné (2010), los procesos de transformación de las potencialidades intelectuales en competencias en áreas concretas (por ejemplo, las enmarcadas en el currículo escolar) implican el componente llamado "inversión", que refiere tanto a aspectos económicos y culturales (por ejemplo, estructura, dinámicas y posibilidades de la escuela y de la familia) como personales (tiempo invertido, perseverancia, motivación, etc.), y en particular, involucra los "catalizadores ambientales" que, a su vez, están directamente relacionados con las condiciones del contexto escolar y familiar.

Los datos de esta investigación corroboran los hallazgos de estudios realizados en el nivel de la educación básica en México, que señalan al contexto socioeducativo (que integra una diversidad de factores económicos, culturales, y de acceso a una diversidad de alternativas educativas no circunscritas necesariamente a la escuela) como un generador de desigualdades en el alumnado y, consecuentemente, como un predictor importante del logro académico, con menor impacto en áreas del conocimiento que depende, en gran medida, de las prácticas pedagógicas y de lo que se pueda hacer, estrictamente, al interior de la escuela.

\section{Conclusiones}

Los modelos jerárquicos multinivel realizados, controlando el efecto del contexto de procedencia, muestran que los desempeños del alumnado en nuestra evaluación de competencia lectora y matemáticas están explicados en mayor medida, por las diferencias individuales. Sin embargo, y pese al efecto de las diferencias individuales, en términos más generales, nuestros datos evidencian que el desempeño en evaluaciones de logro es sensible al tipo de contexto de procedencia. Lamentablemente, tanto en evaluaciones a gran escala de aprendizajes en México, como de habilidades y conocimientos generales que es la prueba PISA, y en las valoraciones que hemos desarrollado en nuestro proyecto, el 
alumnado de escuelas rurales y del sistema indígena es el que presenta los peores resultados de logro.

Aunque no existen medidas directas en la presente investigación del capital cultural de las familias y las escuelas involucradas en el estudio, la caracterización del contexto de procedencia permite suponer la existencia de diferencias marcadas en este sentido, por lo que se han tomado como indicadores del nivel socioeconómico, como expresión de este. El contexto sociodemográfico puede estar incidiendo en el logro, independientemente de la entidad estatal (Morelos o Sonora): en aquellos contextos más desfavorecidos, enmarcados en las zonas rurales donde se ubican las escuelas del sistema indígena y las Telesecundarias en regiones de las etnias indígenas, los indicadores de desempeño académico pueden ser más bajos que en los restantes contextos.

Sin embargo, estos datos descriptivos deben ser tomados con precaución y ahondar en estudios con otras formas de valoración de lo aprendido en el sexto grado de primaria, para ello debe profundizarse en el efecto diferencial de variables personales una vez controlado el efecto que tienen las variables de contexto sobre el logro académico (Cueto et al., 2016; Hernández y Bazán, 2016; Hernández y González-Montesinos, 2011); a través del control que las brechas en los resultados educativos de contextos de procedencia se incrementen debido a que el estudiantado indígena no es evaluado en su primera lengua (Backhoff, Solano, Contreras, Vázquez y Sánchez, 2015; Blanco, 2017).

Asimismo, una limitación de este estudio, que deberá ser mejorada en futuros análisis, es la bondad de ajuste del modelo de validez de constructo para la valoración de los aprendizajes esperados en matemáticas y, con mayor número de tareas o ítems por cada competencia. Como conclusiones derivadas de los resultados de este estudio, se podrían señalar algunos aspectos importantes:

1. El Contexto de Procedencia puede ser determinante tanto de los indicadores de logro académico como del aprendizaje mismo, y del tipo de relaciones entre diferentes factores asociados al logro académico, siempre con ventaja a favor del alumnado de escuelas Privadas; por el contrario, el estudiantado de escuelas o Telesecundarias Rurales en zonas indígenas o del sistema indígena, los que obtienen los peores resultados tanto en Comprensión lectura como en Matemáticas.

2. Los análisis realizados con modelo jerárquico multinivel nos indican que los desempeños de los estudiantes, al término del Sexto Grado de primaria, están explicados en mayor medida por las diferencias individuales, dado que podría darse 
que, en escuelas Privadas, existan escolares que obtuvieron bajos niveles de desempeño, y en escuelas Rurales, existan estudiantes que hayan logrado altos niveles de desempeño.

3. El mayor Grado de Escolaridad del Padre o la Madre predice de manera significativa el logro académico, de manera positiva en Comprensión Lectora y de manera negativa en Matemáticas. Pero, cuando se controla el Contexto de procedencia se incrementa el valor negativo de su efecto, siendo significativo solo en Matemáticas.

4. El Sexo es una variable que predice de manera significativa solamente en Comprensión lectora, cuando se controla el Contexto de Procedencia. Independientemente del Estado y del Contexto de Procedencia, las mujeres pueden obtener mejor logro académico en evaluaciones de Comprensión lectora.

5. Cuando en los modelos de predicción se emplearon las calificaciones anuales obtenidas en las asignaturas de Español y de Matemáticas, se demostró que ambas calificaciones predicen significativamente el logro en Comprensión lectora; sin embargo, en el caso de Matemáticas, solamente la calificación anual en Matemáticas contribuyó de manera relevante.

6. Las estrategias para enfrentar las desigualdades del logro académico, según la variable contexto de origen de las escuelas, implica esfuerzos desde los niveles de las políticas públicas de educación; mejoras en las oportunidades educativas a nivel regional y local; las mejoras a nivel de escuela (en lo tocante a las condiciones básicas para la enseñanza y el aprendizaje, la infraestructura, hasta la gestión escolar); y el fortalecimiento de las habilidades y competencias básicas a nivel del alumnado; la participación de las familias y la comunidad en los procesos educativos; así como el involucramiento del profesorado para mejorar las oportunidades para el aprendizaje del alumnado, por citar algunas estrategias o tomas de acción.

\section{Agradecimientos}

Para realizar esta investigación se obtuvo financiamiento del Consejo Nacional de Ciencia y Tecnología (CONACYT), Ciencia Básica 2010, núm. 151981, cuya vigencia fue del 15 de marzo de 2012 al 15 de abril de 2016.

En esta investigación colaboraron las siguientes personas: Dra. Eneida Ochoa, Maestra Mónica Dávila y Mtro. Rafael Félix, por el Instituto Tecnológico de Sonora; Dra. 
Violeta Fajardo, Psic. Nayely Vega, Mtro, César Hernández, Mtro Aldo Ferrari, Mtro. Néstor

Velarde y Dra. Laura Cruz, por la Universidad Autónoma del Estado de Morelos.

\section{Referencias}

Alanís Herrera, Juan Pablo., Ávila Fernández, Verónica. y Lerma Reyes, Élida. (2009). Las implicaciones de la prueba ENLACE en educación primaria y su relación con el contexto socioeconómico. Red Durango de Investigación Educativa, 1(1), 22-33.

Backhoff Escudero, Eduardo. (2011). La inequidad educativa en México: diferencias en el aprendizaje de la comprensión lectora en educación básica. Profesorado. Revista de curriculum y formación del profesorado, 5(3). Recuperado de: http://www.ugr.es/local/recfpro/rev153ART6.pdf

Backhoff Escudero, Eduardo., Andrade Muñoz, Edgar., Peon Zapata, Margarita., Sánchez Moguel, Andrés. y Bouzas Riaño, Arturo. (2006). El aprendizaje del Español y las Matemáticas en la educación básica en México: sexto de primaria y tercero de secundaria. México: Instituto Nacional para la Evaluación de la Educación.

Backhoff Escudero, Eduardo., Andrade Muñoz, Edgar., Peon Zapata, Margarita. y Sánchez Moguel, Andrés. (2010). Comprensión lectora y habilidades matemáticas de estudiantes de educación básica en México: 2000-2005. Revista Electrónica de Investigación Educativa, 12 (1), 1-15.

Backhoff Escudero, Eduardo., Andrade Muñoz, Edgar., Sánchez Moguel, Andrés. y Peon Zapata, Margarita. (2008). El aprendizaje en tercero de preescolar en México: Lenguaje y comunicación y Pensamiento matemático. México: Instituto Nacional para la Evaluación de la Educación.

Backhoff Escudero, Eduardo., Solano-Flores, Guillermo., Contreras Niño, Luis Ángel., Vázquez Muñoz, Mariana. y Sánchez Moguel, Andrés. (2015). ¿Son adecuadas las traducciones para evaluar los aprendizajes de los estudiantes indígenas? Un estudio con preescolares mayas. Ciudad de México: Instituto Nacional para la Evaluación de la Educación.

Bazán-Ramírez, Aldo., Urbina Pérez, Diana., Domínguez Márquez, Lorena., Mansillas Cervantes, María Isabel. y Gómez Manjarrez, Itzel. (2011). Desarrollo funcional de competencias de producción de textos en alumnos con bajo desempeño en el último año de primaria. Revista Mexicana de Análisis de la Conducta, 37(1), 89-107. doi:10.5514/rmac.v37.i1.24741

Blanco Bosco, Emilio. (2006). Factores escolares asociados a los aprendizajes en la educación primaria mexicana: un análisis multinivel. Revista Iberoamericana sobre Calidad, Eficacia y Cambio en Educación, 6(1), 58-84.

Blanco Bosco, Emilio. (2017). Los alumnos indígenas en México: Siete hipótesis sobre el rezago en los aprendizajes de nivel primario. Revista Iberoamericana sobre Calidad, Eficacia y Cambio en Educación, 15(3), 81-112. 
Carvallo, Mauricio. (2006). Factores que Afectan el Desempeño de los Alumnos Mexicanos en Edad de Educación Secundaria. Un Estudio Dentro de la Corriente de Eficacia Escolar. Revista Iberoamericana sobre Calidad, Eficacia y Cambio en Educación, 4(3), 30-53.

Carvallo, Mauricio., Caso Niebla, Joaquín. y Contreras Niño, Luis Ángel. (2007). Estimación del efecto de variables contextuales en el logro académico de estudiantes de Baja California. REDIE, Revista Electrónica de Investigación Educativa, 9(2). Recuperado de http://redie.uabc.mx/vol9no2/contenido-carvallo.html

Castro, Dina., Lubker, Bobbie., Bryant, Donna. y Skinner, Martie. (2002). Oral language and reading abilities of first-grade Peruvian children: associations with child and family factors. International Journal of Behavioral Development, 26(4), 334-344.

Cervini, Rubén. (2002). Desigualdades socioculturales en el aprendizaje de matemática y lengua de la educación secundaria en Argentina: Un modelo de tres niveles. REDIE, Revista Electrónica de Investigación y Evaluación Educativa, 8(2), 126-148.

Coleman, James S., Campbell, Ernest Q., Hobson, Carol J., McPartland, James., Mood, Alexander M., Weinfeld, Frederic D. y York, Robert L. (1966). Equality of educational opportunity. Washington, D.C.: Oficina de impresión de los Estados Unidos.

Cueto, Santiago., León, Juan., Muñoz, Ismael. y Rosales, Elizabeth. (2016). Conductas, estrategias y rendimiento en lectura en PISA: análisis para el Perú. Revista Iberoamericana sobre Calidad, Eficacia y Cambio en Educación, 14(3), 5-31.

Gagné, Françoys. (2010). Construyendo talentos a partir de la dotación: Breve revisión del MDDT 2.0. En María de los Dolores Valadez Sierra y Silvia Valencia Abundiz (Comp.), Desarrollo y educación del talento en la adolescencia (pp. 64-78). Universidad de Guadalajara: Editorial Universitaria.

Hernández Padilla, Eduardo. (2018). El aprendizaje en escuelas multigrado mexicanas en la prueba PLANEA. Revista Iberoamericana sobre Calidad, Eficacia y Cambio en Educación, 16(3), 123-138.

Hernández Padilla, Eduardo. y Bazán-Ramírez, Aldo. (2016). Efectos Contextuales, Socioeconómicos y Culturales, sobre los Resultados de México en Lectura en PISA 2009. Revista Iberoamericana sobre Calidad, Eficacia y Cambio en Educación, 14(2), 79-95.

Hernández Padilla, Eduardo. y González-Montesinos, Manuel Jorge. (2011). Modelo de ecuación estructural que evalúa las relaciones entre el estatus cultural y económico del estudiante y el logro educativo. REDIE, Revista Electrónica de Investigación Educativa, 13(2), 188-203.

Instituto Nacional para la Evaluación de la Educación [INEE]. (2016). Plan Nacional para la Evaluación de los Aprendizajes (PLANEA). Resultados nacionales 2015 Sexto de primaria y tercero de secundaria. Lenguaje y comunicación y Matemáticas. Recuperado http://planea.sep.gob.mx/content/general/docs/2015/difusion resultados/1 Resultados nacionales Planea 2015.pdf 
Mella, Orlando. y Ortiz, Iván. (1999). Rendimiento escolar. Influencias diferenciales de factores externos e internos. Revista Latinoamericana de Estudios Educativos, 29(1), 69-92.

Reese, Leslie., Mejía Arauz, Rebeca. y Ray Bazán, Antonio. (2012). Mexican parents' and teachers' literacy perspectives and practices: construction of cultural capital. International Journal of Qualitative Studies in Education, 25(8), 983-1003.

Secretaría de Educación Pública. (2013). Resultados Históricos Nacionales 2006-2013. 3ro, 4to, 5to, 6to de Primaria; 1ro, 2do y 3ro de Secundaria. Español, Matemáticas, Formación Cívica $\quad y \quad$ Ética. Recuperado en http://www.enlace.sep.gob.mx/content/gr/docs/2013/historico/00 EB 2013.pdf

Vázquez-Chagoyán, Ricardo. (2005). La escuela a examen. Las reformas educativas más de cuatro décadas de fracasos. Observatorio ciudadano de la educación. Colaboraciones libres. Recuperado https://www.conexiondigital.org/laescuelaaexamen/LA ESCUELA A EXAMEN.pdf 
Revista indizada en

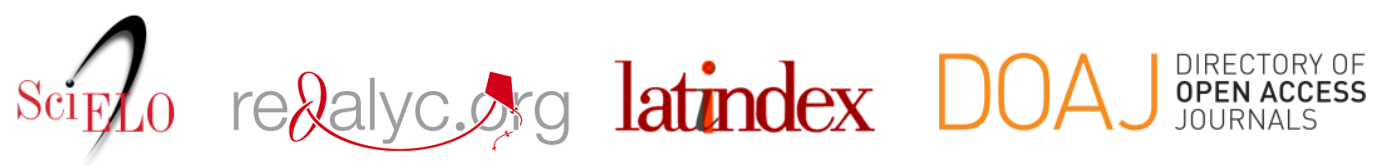

Distribuida en las bases de datos:

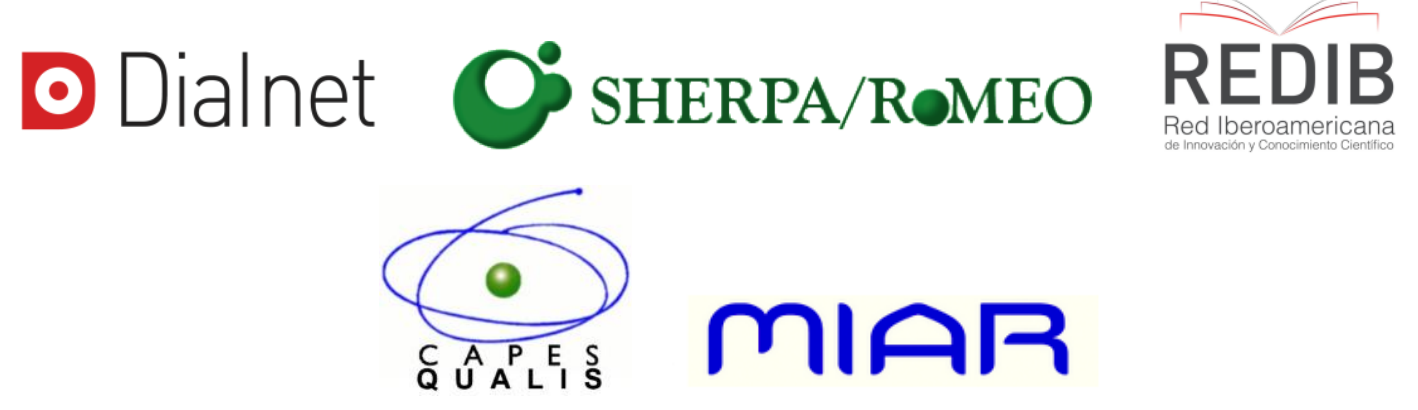

